\title{
THE PRIME $k$-TUPLETS IN ARITHMETIC PROGRESSIONS
}

\author{
By
}

\section{Koichi KAWADA}

\section{§ 1. Introduction and notation.}

In this paper we discuss a problem on the distribution of prime multiplets in arithmetic progressions. Before mentioning our problem we need to introduce the following notation. (In connection with our problem, see also the introduction of Balog's tract [1].)

For an integer $k \geqq 2$, we let $a_{j}(0 \leqq j \leqq k-1)$ be non-zero integers, and let $b_{j}(0 \leqq j \leqq k-1)$ be integers, and put $\boldsymbol{a}=\left(a_{0}, a_{1}, \cdots, a_{k-1}, b_{0}\right), \boldsymbol{b}=\left(b_{1}, \cdots, b_{k-1}\right)$, (Later, we will fix all the coordinates of $\boldsymbol{a}$, and treat an average over $\boldsymbol{b}$. This is why the unsymmetry of the definitions of $\boldsymbol{a}$ and $\boldsymbol{b}$ occurs.),

$$
\begin{aligned}
& R(\boldsymbol{b})=R(\boldsymbol{a}, \boldsymbol{b})=\prod_{j=0}^{k-1}\left|a_{j}\right| \prod_{0 \leq i<j \leq k-1}\left|a_{i} b_{j}-a_{j} b_{\imath}\right|, \\
& N(x ; \boldsymbol{b})=N(x ; \boldsymbol{a}, \boldsymbol{b})=\left\{n ; 1 \leqq a_{j} n+b_{j} \leqq x \text { for all } 0 \leqq j \leqq k-1\right\},
\end{aligned}
$$

and define

$$
\Psi(x ; \boldsymbol{b}, a, q)=\Psi(x ; \boldsymbol{a}, \boldsymbol{b} ; \boldsymbol{a}, q)=\sum_{\substack{n \in N(x ; b) \\ n \equiv a(\bmod q)}} \prod_{j=0}^{k-1} \Lambda\left(a_{j} n+b_{j}\right),
$$

where $\Lambda$ denotes the von Mangoldt function. And, we let, for any prime $p$, $\rho(p)=\rho(p ; \boldsymbol{a}, \boldsymbol{b})$ be the number of solutions of the congruence

$$
\prod_{j=0}^{k-1}\left(a_{j} n+b_{j}\right) \equiv 0 \quad(\bmod p),
$$

and set, if $R(\boldsymbol{b}) \neq 0, \rho(p)<p$ for all prime $p$, and $\left(a_{j} a+b_{j}, q\right)=1$ for all $0 \leqq j \leqq$ $k-1$,

$$
\boldsymbol{\sigma}(\boldsymbol{b} ; a, q)=\sigma(\boldsymbol{a}, \boldsymbol{b} ; a, q)=\frac{1}{q} \prod_{p \mid q}\left(1-\frac{\rho(p)}{p}\right)^{-1} \prod_{p}\left(1-\frac{\rho(p)}{p}\right)\left(1-\frac{1}{p}\right)^{-k}
$$

and $\sigma(b ; a, q)=0$ otherwise. Further, we put

$$
Z(x)=Z(x ; \boldsymbol{a})=\{\boldsymbol{b} ;|N(x ; \boldsymbol{b})| \neq 0\},
$$

where $|N(x ; \boldsymbol{b})|$ denote the length of the interval $N(x ; \boldsymbol{b})$.

Received December 11, 1991, Revised April 15, 1992. 
By a heuristic arguement due to Bateman and Horn [2], it is expected that if $\boldsymbol{\sigma}(\boldsymbol{b} ; a, q) \neq 0$ then

$$
\Psi(x ; \boldsymbol{b}, a, q) \sim \boldsymbol{\sigma}(\boldsymbol{b} ; a, q)|N(x ; \boldsymbol{b})| .
$$

Now we consider the inequality

$$
\sum_{q \leq Q} \max _{1 \leq a \leq q} \sum_{b \in \boldsymbol{Z}(x)}|\Psi(x ; \boldsymbol{b}, a, q)-\boldsymbol{\sigma}(\boldsymbol{b} ; a, q)| N(x ; \boldsymbol{b})|| \ll x^{k}(\log x)^{-\boldsymbol{A}},
$$

for fixed $\boldsymbol{a}$, and for any fixed positive constant $A$. Recently, Maier and Pomerance [3] treated the inequality (1.1), for the case $k=2$, in order to apply their arguement concerning with the difference between consecutive prime numbers, and showed the validity for $Q \leqq x^{\delta}$ with some (small) positive constant $\delta$. Later, Balog [1] proved that the inequality (1.1) holds for the general case $k \geqq 2$, and for a wider range of $Q$, namely $Q \leqq x^{1 / 3}(\log x)^{-B}$ with some positive constant $B$ depending on $A$.

Very recently, Mikawa [4] extend the range of validity of (1.1), for the case $k=2$, to $Q \leqq x^{1 / 2}(\log x)^{-B}$ with some positive constant $B$ depending on $A$, by means of the dispersion method. Mikawa's result seems best possible, for the present, by contrast with the Bombieri-Vinogradov theorem.

In this paper, we give a proof, owing to the traditional circle method, for the validity of $(1.1)$, in the general case $k \geqq 2$, for $Q \leqq x^{1 / 2}(\log x)^{-B}$ with a positive constant $B$ depending on $k$ and $A$.

THEOREM 1. Let $k \geqq 2, \boldsymbol{a}$ and $A>0$ be fixed. Then the inequality (1.1) is valid for

$$
Q \leqq x^{1 / 2}(\log x)^{-B},
$$

where $B$ is some positive constant depending on $k$ and $A$.

Moreover, we shall prove a short interval version of Theorem 1. For $0<$ $y \leqq x$, we reset

$$
\begin{aligned}
& N(x, y ; \boldsymbol{b})=N(x ; \boldsymbol{a}, \boldsymbol{b})=\left\{n ; x-y<a_{j} n+b_{j} \leqq x \text { for all } 0 \leqq j \leqq k-1\right\} \\
& \Psi(x, y ; \boldsymbol{b} ; \boldsymbol{a}, q)=\Psi(x, y ; \boldsymbol{a}, \boldsymbol{b} ; a, q)=\sum_{\substack{n \in N(x, y ; b) \\
n \equiv a(\bmod q)}} \prod_{j=0}^{k-1} \Lambda\left(a_{j} n+b_{j}\right), \\
& Z=Z(x, y ; \boldsymbol{a})=\{\boldsymbol{b} ;|N(x, y ; \boldsymbol{b})| \neq 0\},
\end{aligned}
$$

and write $N=|N(x, y ; \boldsymbol{b})|$ the length of the interval $N(x, y ; \boldsymbol{b})$, for simplicity. Trivially, we see that

$$
N \ll y \quad \text { and } \# Z \ll y^{k-1},
$$

where $\# Z$ means the number of elements of $Z$. 
THEOREM 2. Let $k \geqq 2$, $\boldsymbol{a}$ and $A>0$ be fixed, and assume that

$$
x^{2 / 3}(\log x)^{C_{0}}<y \leqq x,
$$

with some positive constant $C_{0}$ depending on $k$ and $A$. Then we have

$$
\sum_{q \leqq Q} \max _{1 \leqq a \leqq q} \sum_{b \in Z}|\Psi(x, y ; \boldsymbol{b}, a, q)-\sigma(\boldsymbol{b} ; a, q) N| \ll y^{k}(\log x)^{-A},
$$

providing that

$$
Q \leqq y x^{-1 / 2}(\log x)^{-B},
$$

where $B$ is a positive constant depending on $k$ and $A$.

Of course, Theorem 1 is a special case of Theorem 2, so we prove only Theorem 2 in the sequel.

I would like to thank Professor S. Uchiyama for encouragement and for careful reading of the manuscript of this paper. I would also like to thank Dr. H. Mikawa for stimulating discussions and advice.

\section{§2. Preliminaries.}

We use a standard notation in number theory, especially, we denote the greatest common divisor and the least common multiple by (, ) and $[$,$] , res-$ pectively. (We use the square bracket $[$,$] also to denote intervals, but one$ may not be confused.) And throughout the paper, we let $a_{j}(0 \leqq j \leqq k-1)$ be fixed non-zero integers, and let $b_{0}$ be a fixed integer which is prime to $a_{0}$ (if $\left(a_{0}, b_{0}\right)$ $>1$ then our theorem is trivial), and assume that

$$
x^{2 / 3}(\log x)^{3 C+657}<y \leqq x,
$$

with some positive constant $C$. Later, $C$ will be chosen in terms of $k$ and $A$.

Our proof is based on the circle method. We use the functions,

$$
\begin{aligned}
& e(\alpha)=e^{2 \pi i \alpha}, \quad P(\alpha)=P(\alpha ; x, y)=\sum_{x-y<n \leqq x} \Lambda(n) e(n \alpha), \\
& P_{a q}(\alpha)=P_{a q}(\alpha ; x, y)=\sum_{\substack{x-y<a_{0} n+b_{0} \leqq x \\
n \equiv a(\bmod q)}} \Lambda\left(a_{0} n+b_{0}\right) e(n \alpha),
\end{aligned}
$$

and define the major and minor arcs,

$$
\begin{aligned}
& \boldsymbol{M}(c, q)=\left[\frac{c}{q}-\Delta, \frac{c}{q}+\Delta\right], \\
& \boldsymbol{M}=\bigcup_{q \leq Q_{1}} \underset{\substack{1 \leq c \leq q \\
(c, q)=1}}{\bigcup M}(c, q), \\
& \boldsymbol{m}=\left[x^{-1 / 6}, 1+x^{-1 / 6}\right]-\boldsymbol{M}
\end{aligned}
$$


where

$$
Q_{1}=(\log x)^{C}, \quad \Delta=y^{-1}(\log x)^{2 A+2 C(k-1)+2} .
$$

Now we note that $\boldsymbol{M}(c, q)$ 's are disjoint for $q \leqq Q_{1}, 1 \leqq c \leqq q,(c, q)=1$. We also note that if $\boldsymbol{\alpha} \in \boldsymbol{m}$ then there exist co-prime natural numbers $q$ and $c$ such that

$$
q \leqq Q_{1} \text { and } \Delta<\left|\alpha-\frac{c}{q}\right| \leqq q^{-1} x^{-1 / 6}
$$

or

$$
Q_{1}<q \leqq x^{1 / 6} \text { and }\left|\alpha-\frac{c}{q}\right| \leqq q^{-1} x^{-1 / 6}
$$

Our proof is also based on following results.

LEMMA 1. Assume that $\alpha \in \boldsymbol{M}(c, q), q \leqq Q_{1}, 1 \leqq c \leqq q,(c, q)=1$, and write $\alpha=$ $(c / q)+\beta$. Then we have

$$
P(\alpha)=\frac{\mu(q)}{\phi(q)} T(\beta)+O\left(y \exp \left(-\delta_{0}(\log x)^{1 / 5}\right)\right),
$$

where $\delta_{0}$ is a positive constant and $T(\beta)=\sum_{x-y<n \leq x} e(n \beta)$, and as usual, $\phi$ and $\mu$ denote the Euler totient function and the Möbius function, respectively.

LEMMA 2.

$$
\max _{\alpha \in m}|P(\alpha)| \ll y(\log x)^{-C+1}
$$

LEMMA 3. Let

$$
E(x, y ; q)=\max _{\substack{1 \leq a \leq q \\(a, q)=1}} \max _{I \subset[x-y, x]}\left|\sum_{\substack{n \in I \\ n \in a(\bmod q)}} \Lambda(n)-\frac{|I|}{\phi(q)}\right|
$$

where I runs over all intervals in $[x-y, x]$, and $|I|$ denote the length of the interval $I$. Then, for any positive constant $A_{1}$, we have

$$
\sum_{q \leq \tilde{Q}} E(x, y ; q) \ll y(\log x)^{-A_{1}},
$$

where $\tilde{Q}=y x^{-1 / 2}(\log x)^{-B_{1}}$ with a positve constant $B_{1}$ depending on $A_{1}$.

Lemma 1 and Lemma 2 are minor modifications of Pan and Pan [5, Theorem 3 and p. 146]. Their proofs are based on the results about the zeros of Diriclet's $L$ functions, and Lemma 1 is still true for $y>x^{7 / 12+\varepsilon}$ with any positive constant $\varepsilon$, but Lemma 2 holds only for $y$ satisfying (2.1).

Lemma 3 is a Bombieri-Vinogradov theorem for short intervals, and Perelli, Pintz and Salerno [6] proved Lemma 3 for $y>x^{3 / 5+\varepsilon}$ with any $\varepsilon>0$. 


\section{§3. Proof of the Theorem 2.}

At first, we note that we have an admissible bound in the case $\boldsymbol{\sigma}(\boldsymbol{b} ; \boldsymbol{a}, q)=0$. Indeed, if $\left(a_{j} a+b_{j}, q\right)>1$ for some $0 \leqq j \leqq k-1$, or if $\rho(p)=p$ for some prime $p$, then we have $\Psi(x, y ; b ; a, q) \ll(\log x)^{k+1}$. So these cases contribute to the left-hand side of $(1.2)$ at most $O\left(y^{k-1} Q(\log x)^{k+1}\right)$, since the number of elements of $Z$ is $O\left(y^{k-1}\right)$.

As for the case $R(\boldsymbol{b})=0$, we see that the number of $\boldsymbol{b}^{\prime}$ 's is $O\left(y^{k-2}\right)$. Thus, using a trivial bound $\Psi(x, y ; \boldsymbol{b} ; a, q) \ll(y / q)(\log x)^{k}$, this case contributes to the left side of $(1.2)$ at most $O\left(y^{k-1}(\log x)^{k+1}\right)$.

So, in what follows, we consider only the case $\sigma(b, a, q) \neq 0$, that is,

$$
\begin{aligned}
& \left(a_{j} a+b_{j}, q\right)=1 \quad \text { for all } 0 \leqq j \leqq k-1, \\
& \rho(p)<p \quad \text { for all prime number } p, \\
& R(\boldsymbol{b}) \neq 0 .
\end{aligned}
$$

We set $\boldsymbol{\alpha}=\left(\alpha_{1}, \cdots, \alpha_{k-1}\right)$, and

$$
F(\boldsymbol{\alpha})=\prod_{j=1}^{k-1} P\left(\alpha_{j}\right) \cdot P_{a q}\left(-\sum_{j=1}^{k-1} a_{j} \alpha_{j}\right),
$$

then we can write

$$
\begin{aligned}
\Psi(x, y ; \boldsymbol{b} ; a, q) & =\int_{0}^{1} \cdots \int_{0}^{1} F(\boldsymbol{\alpha}) e\left(-\sum_{j=1}^{k-1} b_{j} \alpha_{j}\right) d \alpha_{1} \cdots d \alpha_{k-1} \\
& =I_{M}+\sum_{h=1}^{k-1} I_{m, h}
\end{aligned}
$$

where $I_{M}$ is the integral on the major arcs, and $I_{m, n}$ 's are the integrals on the minor arcs, that is,

$$
I_{M}=\int_{M} \cdots \int_{M} F(\boldsymbol{\alpha}) e\left(-\sum_{j=1}^{k-1} b_{j} \alpha_{j}\right) d \alpha_{1} \cdots d \alpha_{k-1},
$$

and, for $1 \leqq h \leqq k-1$,

$$
I_{m, h}=\int \cdots \int_{\substack{\alpha_{j} \in M(1 \leq j<h) \\ \alpha h \in m \\ \alpha_{j} \in[0,1](h<j \leq k-1)}} F(\boldsymbol{\alpha}) e\left(-\sum_{j=1}^{k-1} b_{j} \alpha_{j}\right) d \alpha_{1} \cdots d \alpha_{k-1} .
$$

In section 4 , we shall prove

$$
S_{m, h}=\sum_{q \leq Q} q \max _{a} \sum_{b \in Z}\left|I_{m, h}\right|^{2} \ll y^{k+1}(\log x)^{-C+2 k+1},
$$

using Lemma 2. Then we have, by Cauchy-Schwartz inequality, 


$$
\begin{aligned}
\sum_{q \leq Q} \max _{a} \sum_{b \in Z}\left|I_{m, h}\right| & \ll\left(\sum_{q \leq Q} \frac{1}{q}\right)^{1 / 2}\left(y^{k-1} S_{m, h}\right)^{1 / 2} \\
& \ll y^{k}(\log x)^{-C / 2+k+1} .
\end{aligned}
$$

Next we turn to $I_{M}$. For $\alpha_{j} \in \boldsymbol{M}\left(c_{j}, q_{j}\right)$, we write $\alpha_{j}=\left(c_{j} / q_{j}\right)+\beta_{j}$, then by Lemma 1,

$$
P\left(\alpha_{j}\right)=\frac{\mu\left(q_{j}\right)}{\phi\left(q_{j}\right)} T\left(\beta_{j}\right)+O\left(y \exp \left(-\delta_{0}(\log x)^{1 / 5}\right)\right) .
$$

We put $\boldsymbol{q}=\left(q_{1}, \cdots, q_{k-1}\right), \boldsymbol{c}=\left(c_{1}, \cdots, c_{k-1}\right), \boldsymbol{\beta}=\left(\boldsymbol{\beta}_{1}, \cdots, \boldsymbol{\beta}_{k-1}\right)$ and

$$
\begin{gathered}
G(\boldsymbol{\beta} ; \boldsymbol{c}, \boldsymbol{q})=\prod_{j=1}^{k-1} T\left(\beta_{j}\right) \cdot P_{a q}\left(-\sum_{j=1}^{k-1} a_{j}\left(\frac{c_{i}}{q_{j}}+\beta_{j}\right)\right), \\
J(\boldsymbol{c}, \boldsymbol{q})=\int \cdots \int_{\mid \boldsymbol{\beta}_{\mid \leq \Delta}} G(\boldsymbol{\beta} ; \boldsymbol{c}, \boldsymbol{q}) e\left(-\sum_{j=1}^{k-1} b_{j} \beta_{j}\right) d \beta_{1} \cdots d \beta_{k-1},
\end{gathered}
$$

where $|\boldsymbol{\beta}| \leqq \Delta$ means $\left|\beta_{j}\right| \leqq \Delta$ for all $1 \leqq j \leqq k-1$. Now we can express

$$
I_{M}=\sum_{q \leq Q_{1}} \prod_{j=1}^{k-1} \frac{\mu\left(q_{j}\right)}{\phi\left(q_{j}\right)} \sum_{c}^{q} e\left(-\sum_{j=1}^{k-1} \frac{c_{j}}{q_{j}} b_{j}\right) \cdot J(\boldsymbol{c}, \boldsymbol{q})+O\left(\frac{y}{q} \exp \left(-\delta_{1}(\log x)^{1 / 5}\right)\right),
$$

where $\delta_{1}$ is a positive constant and

$\boldsymbol{q} \leqq Q_{1}$ means $q_{j} \leqq Q_{1}$ for all $1 \leqq j \leqq k-1$,

$\sum_{c}^{q} *$ means the summation over all $c$ such that every coordinate

$c_{j}$ is prime to $q_{j}$, and $1 \leqq c_{j} \leqq q_{j}$.

Morover, we write $J(\boldsymbol{c}, \boldsymbol{q})=J_{0}(\boldsymbol{c}, \boldsymbol{q})-\sum_{h=1}^{k-1} J_{h}(\boldsymbol{c}, \boldsymbol{q})$, where

$$
J_{0}(\boldsymbol{c}, \boldsymbol{q})=\int_{0}^{1} \cdots \int_{0}^{1} G(\boldsymbol{\beta} ; \boldsymbol{c}, \boldsymbol{q}) e\left(-\sum_{j=1}^{k-1} b_{j} \beta_{j}\right) d \beta_{1} \cdots d \beta_{k-1},
$$

and, for $1 \leqq h \leqq k-1$,

$$
J_{h}(\boldsymbol{c}, \boldsymbol{q})=\int \cdots \int_{\substack{\beta_{j} \in[0,1](1 \leq j<h) \\ \beta_{h \in[\Delta, 1-4]} \in \beta_{j} \leq \dot{\Delta}(h<j \leq k-1)}} G(\boldsymbol{\beta} ; \boldsymbol{c}, \boldsymbol{q}) e\left(-\sum_{j=1}^{k-1} b_{j} \beta_{j}\right) d \beta_{1} \cdots d \beta_{k-1} .
$$

In section 5 , we will show that, for $1 \leqq h \leqq k-1$,

$$
\sum_{d \in Z}\left|J_{h}(\boldsymbol{c}, \boldsymbol{q})\right|^{2} \ll \frac{y^{k}}{q^{2}} \Delta^{-1},
$$

and that

$$
\begin{aligned}
J_{0}(\boldsymbol{c}, \boldsymbol{q})= & \frac{\left|a_{0}\right| N}{\boldsymbol{\phi}\left(\left|a_{0}\right|[q, r]\right)} \sum_{\boldsymbol{d}}^{q} e^{\prime}\left(-\sum_{j=1}^{k-1} \frac{c_{j}}{q_{j}} a_{j} d_{j}\right) \\
& +O\left(\left(E\left(x, y ;\left|a_{0}\right|[q, r]\right)+1\right)(\log x)^{k+1}\right),
\end{aligned}
$$


where $r$ denotes the least common multiple of the coordinates of $\boldsymbol{q}$, and $\boldsymbol{d}=$ $\left(d_{1}, \cdots, d_{k-1}\right)$, and $\sum_{d}^{q} \#$ denotes the summation over $d_{j}$ 's satisfying the conditions

$$
\begin{gathered}
1 \leqq d_{j} \leqq q_{j} \quad \text { for all } 1 \leqq j \leqq k-1, \\
a \equiv d_{j}\left(\bmod \left(q, q_{j}\right)\right) \quad \text { for all } 1 \leqq j \leqq k-1, \\
d_{i} \equiv d_{j}\left(\bmod \left(q_{i}, q_{j}\right)\right) \quad \text { for all } 1 \leqq i<j \leqq k-1,
\end{gathered}
$$

and $\left(a_{0} d_{j}+b_{0}, q_{j}\right)=1$ for all $1 \leqq j \leqq k-1$.

(3.8) yields

$$
\begin{aligned}
& \sum_{q \leq Q} \max _{a} \sum_{\boldsymbol{b} \in Z}\left|\prod_{\boldsymbol{q} \leq Q_{1}} \prod_{j=1}^{k-1} \frac{\mu\left(q_{j}\right)}{\phi\left(q_{j}\right)} \sum_{\boldsymbol{c}}^{q} * e\left(-\sum_{j=1}^{k-1} \frac{c_{j}}{q_{j}} b_{j}\right) \sum_{h=1}^{k-1} J_{h}(\boldsymbol{c}, \boldsymbol{q})\right| \\
\ll & \left(\sum_{q \leq Q} \frac{1}{q}\right)^{1 / 2}\left(y^{k-1} Q_{1}{ }^{k-1} \sum_{q \leq Q} q \max _{a} \sum_{h=1}^{k-1} \sum_{\boldsymbol{q}} \prod_{j=1}^{k-1} \phi\left(q_{j}\right)^{-1} \sum_{\boldsymbol{c}} \sum_{\boldsymbol{b}}\left|J_{h}\right|^{2}\right)^{1 / 2} \\
(3.10) \quad & \ll y^{k}(\log x)^{-A} .
\end{aligned}
$$

By (3.7), (3.9) and (3.10), we have

$$
\begin{aligned}
& \sum_{q \leq Q} \max _{a} \sum_{b \in Z}\left|I_{M}-\sigma(\boldsymbol{b} ; a, q) N\right| \\
& =\sum_{q \leq Q} \max _{a} \sum_{b \in Z}|S(\boldsymbol{b} ; a, q)| a_{0}|N-\sigma(\boldsymbol{b} ; a, q) N| \\
& \quad+O\left((\log x)^{k+1}\left(y^{k-1} Q Q_{1}^{k-1}+\sum_{q \leq Q} \sum_{q \leq Q_{1}} E\left(x, y ;\left|a_{0}\right|[q, r]\right)\right)\right) \\
& \quad+O\left(y^{k}(\log x)^{-A}+y^{k} \exp \left(-\delta_{1}(\log x)^{1 / 5}\right)(\log x)\right)
\end{aligned}
$$

where

$$
S(\boldsymbol{b} ; a, q)=\sum_{q \leq Q_{1}} \frac{1}{\phi\left(\left|a_{0}\right|[q, r]\right)} \prod_{j=1}^{k-1} \frac{\mu\left(q_{j}\right)}{\phi\left(q_{j}\right)} \sum_{\boldsymbol{d}}^{q} \prod_{j=1}^{k-1} c_{q_{j}}\left(a_{j} d_{j}+b_{j}\right)
$$

and $c_{q}(n)=\sum_{\substack{m=1 \\(m, q)=1}}^{q} e\left(\frac{m}{q} n\right)$ is the Ramanujan sum.

In secton 6 , we shall prove

$$
\begin{aligned}
S(\boldsymbol{b} ; a, q)= & \frac{1}{\left|a_{0}\right|} \boldsymbol{\sigma}(\boldsymbol{b} ; a, q) \\
& +O\left(\frac{1}{q} \tau_{K}(q) \tau_{K}(R(\boldsymbol{b}))(\log x)^{-C+1}\right),
\end{aligned}
$$

of course, on (3.1), (3.2) and (3.3). Here $K$ is a natural number depending only on $k$, and $\tau_{K}(m)$ is the number of ways of writing $m$ as a product of $K$ factors, the order of the factors being taken account. It follows, by known results about divisor functions, that 


$$
\sum_{q \leq Q} \frac{\tau_{K}(q)}{q} \ll(\log Q)^{K}, \text { and } \sum_{\substack{b \in Z \\ R(b) \neq 0}} \tau_{K}(R(b)) \ll y^{k-1}(\log x)^{K_{1}},
$$

with a constant $K_{1}$ depending only on $k$.

Then the first term of (3.11) contributes

$$
\ll y^{k}(\log x)^{-C+K_{2}},
$$

where $K_{2}$ is a constant depending only on $k$.

Estimation of the second term of (3.11), of course, relies on Lemma 3. It follows that

$$
\sum_{q \leq Q} \sum_{q \leq Q_{1}} E\left(x, y:\left|a_{0}\right|[q, r]\right) \ll Q_{1}^{2 k} \sum_{m \leq\left|a_{0}\right| Q Q_{1}^{k-1}} E(x, y ; m)
$$

providing that $\left|a_{0}\right| Q Q_{1}^{k-1} \leqq \tilde{Q}$, that is,

$$
Q \leqq y x^{-1 / 2}(\log x)^{-B} .
$$

Here $\tilde{Q}$ corresponds to $A_{1}=A+3 k+1$ in (2.2) of Lemma 3 , and $B$ is a constant depending on $A$ and $k$. We observe that any other terms is admissible only if $Q \leqq y(\log x)^{-B_{0}}$ with some constant $B_{0}$.

Hence, Theorem 1 follows from (3.4), (3.7), (3.11), (3.13) and (3.14) with a suitable choice of $C$, under assumption of (3.5), (3.8), (3.9) and (3.12).

\section{§4. Estimation of $S_{m, h}$.}

In this section, we prove (3.5). We use Bessel's inequality repeatedly to obtain

$$
\begin{aligned}
\sum_{b \in Z}\left|I_{m, h}\right|^{2} & =\sum_{b_{1}} \cdots \sum_{b_{k-1}}\left|\int P\left(\alpha_{1}\right)\left(\int \cdots \int \cdots d \alpha_{2} \cdots d \alpha_{k-1}\right) e\left(-b_{1} \alpha_{1}\right) d \alpha_{1}\right|^{2} \\
& \leqq \int\left|P\left(\alpha_{1}\right)\right|^{2} \sum_{b_{2}} \cdots \sum_{b_{k-1}}\left|\int P\left(\alpha_{2}\right)\left(\int \cdots \cdots d \alpha_{k-1}\right) e\left(-b_{2} \alpha_{2}\right) d \alpha_{2}\right|^{2} d \alpha_{1} \\
& \leqq \cdots \cdots \\
& \leqq \int \cdots \int_{\substack{\alpha_{h} \in m \\
\alpha_{j \in M} \in(1 \leq j<h) \\
\alpha_{j} \in[0,1](k<j \leq k-1)}}\left(\prod_{j=1}^{k-1}\left|P\left(\alpha_{j}\right)\right|^{2}\right)\left|P_{a q}\left(-\sum_{j=1}^{k-1} a_{j} \alpha_{j}\right)\right|^{2} d \alpha_{1} \cdots d \alpha_{k-1} \\
& \leqq \int_{m}\left|P\left(\alpha_{h}\right)\right|^{2} U_{h} d \alpha_{h},
\end{aligned}
$$

where, for $k \geqq 3$, 


$$
\begin{aligned}
& U_{h}=\int_{0}^{1} \cdots \int_{0}^{1} \prod_{\substack{j=1 \\
j \neq h}}^{k-1}\left|P\left(\alpha_{j}\right)\right|^{2}\left|P_{a q}\left(-\sum_{j=1}^{k-1} a_{j} \alpha_{j}\right)\right|\left(\prod_{\substack{j=1 \\
j \neq h}}^{k-1} d \alpha_{j}\right) \\
& =\sum_{\substack{x-y<a_{0} m_{1}+b_{0}, a_{0} m_{2}+b_{0} \leq x \\
m_{1} \equiv m_{2} \equiv a\left(\bmod _{q}\right)}} \Lambda\left(a_{0} m_{1}+b_{0}\right) \Lambda\left(a_{0} m_{2}+b_{0}\right) e\left(-a_{h} \alpha_{h}\left(m_{1}-m_{2}\right)\right)
\end{aligned}
$$

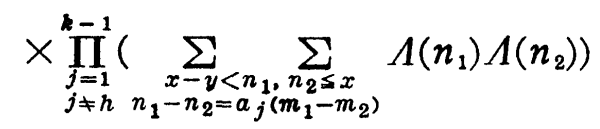

$$
\begin{aligned}
& =\sum_{\substack{|r| \leq y \\
r=0(\bmod q)}} e\left(-a_{h} \alpha_{h} r\right) \sum_{\substack{x-y<a_{0} m+b_{0} \leq x \\
m=a(\bmod q) \\
x-y<a_{0}(m-r)+b_{0} \leq x}} \Lambda\left(a_{0} m+b_{0}\right) \Lambda\left(a_{0}(m-r)+b_{0}\right) \\
& \times \sum_{\substack{j=1 \\
j \neq h}}^{k-1}\left(\sum_{\substack{x-y<n \leq x \\
x-y<n-a_{j} r \leq x}} \Lambda(n) \Lambda\left(n-a_{j} r\right)\right) \\
& =\sum_{\substack{1 r \mid \leq y \\
r \equiv 0(\bmod q)}} e\left(-a_{h} \alpha_{h} r\right) R_{h}(r ; a, q), \quad \text { say, }
\end{aligned}
$$

and, for $k=2$,

$$
\begin{aligned}
& U_{1}=\left|P_{a q}\left(-a_{1} \alpha_{1}\right)\right|^{2} \\
& =\sum_{\substack{x-y<a_{0} m_{1}+b_{0}, a_{0} m_{1} m_{1}=m_{2} \equiv a(\bmod q) \\
m_{1} \leq b_{0} \leq x}} \Lambda\left(a_{0} m_{1}+b_{0}\right) \Lambda\left(a_{0} m_{2}+b_{0}\right) e\left(-a_{1} \alpha_{1}\left(m_{1}-m_{2}\right)\right) \\
& =\sum_{\substack{r \mid r \leq y \\
r \equiv 0(\bmod q)}} e\left(-a_{1} \alpha_{1} r\right) \sum_{\substack{x-y<a_{0} m+b_{0} \leq x \\
m \neq a\left(m o d \\
x-y<a_{0}(m-r)+b_{0} \leq x\right.}} \Lambda\left(a_{0} m+b_{0}\right) \Lambda\left(a_{0}(m-r)+b_{0}\right) \\
& =\sum_{\substack{1, r \mid \leq y \\
r \equiv 0(\bmod q)}} e\left(-a_{1} \alpha_{1} r\right) R_{1}(r ; a, q), \quad \text { say. }
\end{aligned}
$$

Trivially, we have

$$
R_{h}(r ; q, a) \ll y^{k-1} q^{-1}(\log x)^{2 k-2},
$$

for both cases $k=2$ and $k \geqq 3$. By (4.1), (4.2), (4.3) and (4.4),

$$
\begin{aligned}
& S_{m, h} \leqq \sum_{q \leq Q} q \max _{a} \sum_{\substack{1 \\
r=0(\bmod q)}} R_{h}(r ; q, a) \int_{m}\left|P\left(\alpha_{h}\right)\right|^{2} e\left(-a_{h} \alpha_{h} r\right) d \alpha_{h} \\
& \left.\ll y^{k-1}(\log x)^{2 k-2} \sum_{q \leq Q} \sum_{\substack{1, y \leq y \\
r \equiv 0(\bmod q)}}\left|\int_{m}\right| P(\alpha)\right|^{2} e\left(-a_{h} \alpha r\right) d \alpha \mid \\
& \left.\ll y^{k-1}(\log x)^{2 k-2} \sum_{0<|r| \leq y} \tau(|r|)\left|\int_{m}\right| P(\alpha)\right|^{2} e\left(-a_{n} \alpha r\right) d \alpha \mid \\
& +y^{k-1} Q(\log x)^{2 k-2} \int_{0}^{1}|P(\alpha)|^{2} d \alpha,
\end{aligned}
$$

where $\tau$ denotes the divisor function. It is easy to see that 


$$
\int_{0}^{1}|P(\alpha)|^{2} d \alpha=\sum_{x-y<n \leq x} \Lambda(n)^{2} \ll y(\log x)
$$

and

$$
\sum_{0<|r| \leq y} \tau(|r|)^{2} \ll y(\log x)^{3} .
$$

So the second term of (4.5) is admissible in (3.5), and the sum in the first term of (4.5) contributes

$$
\begin{aligned}
& \ll\left(\sum_{0<|r| \leq y} \tau(|r|)^{2}\right)^{1 / 2}\left(\left.\left.\sum_{0<|r| \leq y}\left|\int_{m}\right| P(\alpha)\right|^{2} e\left(-a_{h} \alpha r\right) d \alpha\right|^{2}\right)^{1 / 2} \\
& \ll\left(y(\log x)^{3} \int_{m}|P(\alpha)|^{4} d \alpha\right)^{1 / 2} \\
& \ll\left(y(\log x)^{3} \max _{\alpha \in m}|P(\alpha)|^{2} \int_{0}^{1}|P(\alpha)|^{2} d \alpha\right)^{1 / 2} \\
& \ll y^{2}(\log x)^{-C+3}
\end{aligned}
$$

by virtue of a bound of Lemma 2. Now we obtain (3.5).

\section{§ 5. Evaluation of $J_{h}(\boldsymbol{c}, \boldsymbol{q})$.}

At first, we prove (3.8). It is well known that $|T(\beta)| \ll\|\beta\|^{-1}$, where $\|\beta\|$ denote the distance detween $\beta$ and the nearest integer of it, as usual. So we get

$$
\int_{\Delta}^{1-\Delta}|T(\beta)|^{2} d \beta \ll \int_{\Delta}^{1 / 2} \beta^{-2} d \beta \ll \Delta^{-1}
$$

Then, for $1 \leqq h \leqq k-1$, we repeat using Bessel's inequality, similarly to (4.1), to obtain

$$
\begin{aligned}
\sum_{\boldsymbol{b} \in Z}\left|J_{h}(\boldsymbol{c}, \boldsymbol{q})\right|^{2} & \\
& \ll \int \cdots \int_{j=1}^{k-1}\left|T\left(\beta_{j}\right)\right|^{2} \cdot\left|P_{a q}\left(-\sum_{j=1}^{k-1} a_{j}\left(\frac{c_{j}}{q_{j}}+\beta_{j}\right)\right)\right|^{2} d \beta_{1} \cdots d \beta_{k-1} \\
& \ll\left(\frac{y}{q}\right)^{2} \prod_{\substack{j=1 \\
j \neq h}}^{k-1}\left(\int_{0}^{1}\left|T\left(\beta_{j}\right)\right|^{2} d \beta_{j}\right) \cdot \int_{\Delta}^{1-\Delta}\left|T\left(\beta_{h}\right)\right|^{2} d \beta_{h} \\
& \ll \frac{y^{k}}{q^{2}} \Delta^{-1} .
\end{aligned}
$$

as required in (3.8).

Next we turn to prove (3.9). Caluculating the integrals about $\beta_{j}$ 's, we see

$$
J_{0}(\boldsymbol{c}, \boldsymbol{q})=\sum_{\substack{n \in N \\ n \equiv \boldsymbol{a}(\boldsymbol{x}, y ; b)}} \Lambda\left(a_{0} n+b_{0}\right) e\left(-\sum_{j=1}^{k-1} \frac{c_{j}}{q_{j}} a_{j} n\right) .
$$

We devide the above sum about residue classes of $n$ to moduli $q_{j}$ 's, and write 


$$
J_{0}(\boldsymbol{c}, \boldsymbol{q})=\sum_{\boldsymbol{d}}^{q} e\left(-\frac{c_{j}}{q_{j}} a_{j} d_{j}\right) V(\boldsymbol{d}, \boldsymbol{q}),
$$

where $\boldsymbol{d}=\left(d_{1}, \cdots, d_{k-1}\right), \sum_{d}^{q}$ means the summation over all $d_{j}$ 's satisfying $1 \leqq$ $d_{j} \leqq q_{j}$, and

Unless

$$
V(\boldsymbol{d}, \boldsymbol{q})=\sum_{\substack{n \in N(x, y: b) \\ n \equiv a(\bmod q) \\ n \equiv d\left(\bmod q q_{j}\right)(1 \leqq j \leqq k-1)}} \Lambda\left(a_{0} n+b_{0}\right)
$$

$$
\begin{gathered}
a \equiv d_{j}\left(\bmod \left(q, q_{j}\right)\right) \quad \text { for all } 1 \leqq j \leqq k-1, \\
d_{i} \equiv d_{j}\left(\bmod \left(q_{i}, q_{j}\right)\right) \quad \text { for all } 1 \leqq i<j \leqq k-1,
\end{gathered}
$$

the sum $V(\boldsymbol{d}, \boldsymbol{q})$ is empty. And unless

$$
\left(a_{0} d_{j}+b_{0}, q_{j}\right)=1 \quad \text { for all } 1 \leqq j \leqq k-1,
$$

plainly, we get $V(\boldsymbol{d}, \boldsymbol{q})=O\left((\log x)^{2}\right)$.

If the conditions (5.2), (5.3) and [5.4) are satisfied, there is an integer $M=$ $M(\boldsymbol{d}, \boldsymbol{q} ; a, q)$ such that the congruence conditions apearing in the summation of $V(\boldsymbol{d}, \boldsymbol{q})$ are equivalent to

$$
n \equiv M(\bmod [q, r])
$$

and $\left(a_{0} M+b_{0},[q, r]\right)=1$. Here $r=\left[q_{1}, \cdots, q_{k-1}\right]$, that is, the least common multiple of the all coordinates of $\boldsymbol{q}$, as mentioned in section 3 . Thus we can write

$$
\begin{aligned}
V(\boldsymbol{d}, \boldsymbol{q}) & =\sum_{\substack{n \in N(x ; y ; \boldsymbol{b}) \\
n \equiv M(\bmod [q, r])}} \Lambda\left(a_{0} n+b_{0}\right) \\
& =\sum_{\substack{\left(m-b_{0}\right) / a_{0} \in N(x, y ; \boldsymbol{b}) \\
m \equiv a_{0} M+b_{0}\left(\bmod \left|a_{0}\right|[q, r]\right)}} \Lambda(m) \\
& =\frac{1}{\phi\left(\left|a_{0}\right|[q, r]\right)}\left|a_{0}\right| N+O\left(E\left(x, y ;\left|a_{0}\right|[q, r]\right)\right) .
\end{aligned}
$$

These evaluations with (5.1) yield (3.9).

\section{§ 6. Calculation of the singular series $S(b ; a, q)$.}

In this section, we prove (3.12). We write

$$
\begin{aligned}
S(\boldsymbol{b} ; a, q) & =\sum_{r \leq Q_{1}} \frac{\mu(r)^{2}}{\phi\left(\left|a_{0}\right|[q, r]\right)} W(r)+\sum_{Q_{1} \leq r \leq Q_{1}^{k-1}} \frac{\mu(r)^{2}}{\phi\left(\left|a_{0}\right|[q, r]\right)} W_{1}(r) \\
& =S_{1}+S_{2}, \quad \text { say, }
\end{aligned}
$$

where 


$$
W(r)=\sum_{[\boldsymbol{q}]=r} \prod_{j=1}^{k-1} \frac{\mu\left(q_{j}\right)}{\phi\left(q_{j}\right)} \sum_{d}^{q} \prod_{j=1}^{k-1} c_{q_{j}}\left(a_{j} d_{j}+b_{j}\right)
$$

and $W_{1}(r)$ is the sum with the condition $q \leqq Q_{1}$ added to the above sum. The symbol $[\boldsymbol{q}]=r$ means that the least common multiple of the all coordinates of $\boldsymbol{q}$ is $r$.

We can see that $W(r)$ is multiplicative by a simple arithmatical deduction. Indeed, for $[\boldsymbol{q}]=r=r_{1} r_{2},\left(r_{1}, r_{2}\right)=1$, we put $q_{j}^{(i)}=\left(q_{j}, r_{i}\right)$ and $\boldsymbol{q}_{i}=\left(q_{1}^{(i)}, \cdots, q_{k-1}^{(i)}\right)$ for $i=1,2,1 \leqq j \leqq k-1$. Then this correspondence between $\boldsymbol{q}^{\prime} \mathbf{s}$ satisfying $[\boldsymbol{q}]=r$ and pairs $\left(\boldsymbol{q}_{1}, \boldsymbol{q}_{2}\right)$ satisfying $\left[\boldsymbol{q}_{i}\right]=r_{i}(i=1,2)$ is one-to-one. Moreover, we can set $d_{j}=e_{j}^{(1)} q_{j}^{(2)}+e_{j}^{(2)} q_{j}^{(1)}$, where $e_{j}^{(i)}$ runs through residue classes of modulo $q_{j}^{(i)}$, for $i=1,2,1 \leqq j \leqq k-1$. We have, for $1 \leqq i, j \leqq k-1$,

$$
\begin{aligned}
& d_{j} \equiv a\left(\bmod \left(q_{j}, q\right)\right) \Longleftrightarrow e_{j}^{(1)} q_{j}^{(2)} \equiv a\left(\bmod \left(q j^{(1)}, q\right)\right) \text { and } e_{j}^{(2)} q_{j}^{(1)} \equiv a\left(\bmod \left(q_{j}^{(2)}, q\right)\right), \\
& d_{i} \equiv d_{j}\left(\bmod \left(q_{i}, q_{j}\right)\right) \Longleftrightarrow e_{i}^{(1)} q_{i}^{(2)} \equiv e_{j}^{(1)} q_{j}^{(2)}\left(\bmod \left(q_{i}^{(1)}, q_{j}^{(1)}\right)\right) \\
& \text { and } e_{i}^{(2)} q_{i}^{(1)} \equiv e_{j}^{(2)} q_{j}^{(1)}\left(\bmod \left(q_{i}^{(2)}, q_{j}^{(2)}\right)\right), \\
&\left(a_{0} d_{j}+b_{0}, q_{j}\right)=1 \Longleftrightarrow\left(a_{0} e_{j}^{(1)} q_{j}^{(2)}+b_{0}, q_{j}^{(1)}\right)=1 \text { and }\left(a_{0} e_{j}^{(2)} q_{j}^{(1)}+b_{0}, q_{j}^{(2)}\right)=1 .
\end{aligned}
$$

Now we write $d_{j}^{(1)}=e_{j}^{(1)} q_{j}^{(2)}, d_{j}^{(2)}=e_{j}^{(2)} q_{j}^{(1)}, \boldsymbol{d}_{i}=\left(d_{1}^{(i)}, \cdots, d_{k-1}^{(i)}\right)$ for $i=1,2$, and get

$$
\begin{aligned}
W\left(r_{1} r_{2}\right)= & \sum_{\left[q_{1}\right]=r_{1}} \sum_{\left[q_{2}\right]=r_{2}} \prod_{j=1}^{k-1}\left(\frac{\mu\left(q_{j}^{(1)}\right)}{\phi\left(q_{j}^{(1)}\right)} \frac{\mu\left(q_{j}^{(2)}\right)}{\phi\left(q_{j}^{(2)}\right)}\right) \\
& \times \sum_{d_{1}}^{q_{1}} \sum_{d_{2}}^{q_{2}} \prod_{j=1}^{k-1}\left(c_{q_{j}^{(1)}}\left(a_{j} d_{j}^{(1)}+b_{j}\right) c_{q_{j}^{(2)}}\left(a_{j} d_{j}^{(2)}+b_{j}\right)\right) \\
= & W\left(r_{1}\right) W\left(r_{2}\right) .
\end{aligned}
$$

Next, we attend to $W(p)$ for a prime number $p$. If $[\boldsymbol{q}]=p$ then $q_{j}=1$ or $p$ for all $1 \leqq j \leqq k-1$, and at least one $q_{j}$ is $p$. We denote by $M$ the set of subscript of $q_{j}$ 's such that $q_{j}=p$. Then,

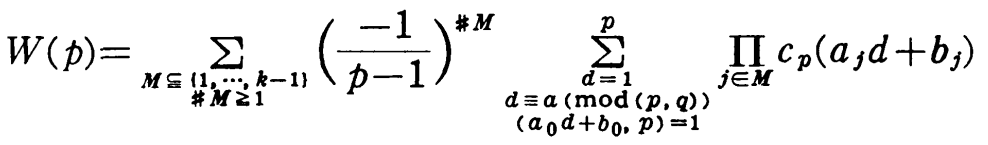

$$
\begin{aligned}
& =\sum_{\substack{d=1 \\
d \equiv a(\bmod (p, q)) \\
\left(a_{0} d+b_{0}, p\right)=1}}^{p}\left(\sum_{M \in(1, \cdots, k-1,} \prod_{j \in \mathcal{M}}\left(\frac{-c_{p}\left(a_{j} d+b_{j}\right)}{p-1}\right)-1\right) \\
& =\sum_{\substack{d=1 \\
d \equiv a(p, q) \\
\left(a_{0} d+b_{0}, p\right)=1}}^{p}\left(\prod_{j=1}^{k-1}\left(1-\frac{c_{p}\left(a_{j} d+b_{j}\right)}{p-1}\right)-1\right),
\end{aligned}
$$


where $\# M$ denote the number of elements of $M$. Therefore, noticing that (3.1), (3.2) and (3.3), we obtain

$$
W(p)= \begin{cases}\left(1-\frac{1}{p}\right)^{-k+1}-1 & \text { (if } p \mid q) \\ (p-\rho(p))\left(1-\frac{1}{p}\right)^{-k+1}-p & \text { (if } \left.p \nmid q \text { and } p \mid a_{0}\right), \\ (p-\rho(p))\left(1-\frac{1}{p}\right)^{-k+1}-p+1 & \text { (if } \left.p \nmid q \text { and } p \nmid a_{0}\right)\end{cases}
$$

and

$$
\begin{aligned}
& \frac{\mu(r)^{2}}{\phi\left(\left|a_{0}\right|[q, r]\right)} W(r)=\frac{1}{\left|a_{0}\right| q} \prod_{p \mid\left(a_{0} q\right)}\left(1-\frac{1}{p}\right)^{-1} \prod_{\substack{p|r \\
p| q}}\left(\left(1-\frac{1}{p}\right)^{-k+1}-1\right) \\
& \quad \times \prod_{\substack{p \mid r \\
p \nmid q \\
p \nmid a_{0}}}\left(\left(1-\frac{\rho(p)}{p}\right)\left(1-\frac{1}{p}\right)^{-k+1}-1\right) \prod_{\substack{p \mid r \\
p \nmid q \\
p \nmid a_{0}}}\left(\left(1-\frac{\rho(p)}{p}\right)\left(1-\frac{1}{p}\right)^{-k}-1\right) .
\end{aligned}
$$

Further $p \nmid R(\boldsymbol{b})$ implies $\rho(p)=k$, so

$$
\left(1-\frac{\rho(p)}{p}\right)\left(1-\frac{1}{p}\right)^{-k}-1 \ll p^{-2},
$$

and, for $p \mid R(\boldsymbol{b})$, the above term is $\ll 1 / p$, where the implied constants depend only on $k$. Plainly, we also get

$$
\left(1-\frac{1}{p}\right)^{-k+1}-1 \ll \frac{1}{p}, \quad\left(1-\frac{\rho(p)}{p}\right)\left(1-\frac{1}{p}\right)^{-k+1}-1 \ll \frac{1}{p},
$$

with the implied constants depending only on $k$. These inequalities and (6.2) shows

$$
\begin{aligned}
\left|\frac{\mu(r)^{2}}{\phi\left(\left|a_{0}\right|[q, r]\right)} W(r)\right| & \leqq \frac{1}{\left|a_{0}\right| q} \prod_{p \mid\left(a_{0} q\right)} 2 \prod_{\substack{p \mid r \\
p !\left(a_{0} \mid r R(b)\right)}} \frac{L}{p} \prod_{\substack{p \mid r \\
p \times\left(a_{0} q R(b)\right)}} \frac{L}{p^{2}} \\
& \leqq \frac{\tau\left(\left|a_{0}\right| q\right)}{\left|a_{0}\right| q}\left(r,\left|a_{0}\right| q R(\boldsymbol{b})\right) \frac{\tau_{L}(r)}{r^{2}}
\end{aligned}
$$

where $L$ is a (sufficiently large) natural number which depends only on $k$. It is known about the divisor function $\tau_{L}(r)$ that

$$
\sum_{r \leq t} \tau_{L}(r) \ll t(\log t)^{L-1}
$$

for $t \geqq 2$, so we have, by partial summation,

$$
\sum_{r>Q_{1}} \frac{\mu(r)^{2}}{\phi\left(\left|a_{0}\right|[q, r]\right)} W(r) \ll \frac{\tau_{K}(q)}{q} \tau_{K}(R(\boldsymbol{b})) \frac{1}{Q_{1}}\left(\log Q_{1}\right)^{L-1} .
$$

with $K$ depending only on $k$. And then, by (6.2), 


$$
\begin{aligned}
\sum_{r=1}^{\infty} \frac{\mu(r)^{2}}{\phi\left(\left|a_{0}\right|[q, r]\right)} W(r) \\
=\frac{1}{\left|a_{0}\right| q} \prod_{p_{\mid}\left(a_{0} q\right)}\left(1-\frac{1}{p}\right)^{-1} \prod_{p \mid q}\left(1-\frac{1}{p}\right)^{-k+1} \sum_{\substack{p \nmid q \\
p \nmid a_{0}}}\left(1-\frac{\rho(p)}{p}\right)\left(1-\frac{1}{p}\right)^{-k+1} \\
\quad \times \prod_{\substack{p \nmid q \\
p \nmid a_{0}}}\left(1-\frac{\rho(p)}{p}\right)\left(1-\frac{1}{p}\right)^{-k} \\
=\frac{1}{\left|a_{0}\right| q} \prod_{p \mid q}\left(1-\frac{1}{p}\right)^{-k} \prod_{p \nmid q}\left(1-\frac{\rho(p)}{p}\right)\left(1-\frac{1}{p}\right)^{-k} \\
=\frac{1}{\left|a_{0}\right|} \sigma(b ; a, q) .
\end{aligned}
$$

Thus, with (6.4), we have

$$
S_{1}=\frac{1}{\left|a_{0}\right|} \sigma(b ; a, q)+O\left(\frac{\tau_{K}(q)}{q} \tau_{K}(R(b))(\log x)^{-C+1}\right) .
$$

Finally, we estimate $S_{2}$. We let

$$
W_{2}(r)=\sum_{[q]=r} \prod_{j=1}^{k-1} \frac{\mu\left(q_{j}\right)^{2}}{\phi\left(q_{j}\right)}\left|\sum_{d}^{q} \prod_{j=1}^{k-1} c_{q_{j}}\left(a_{j} d_{j}+b_{j}\right)\right|,
$$

then we can see, at once, that $W_{2}(r)$ is multiplicative by comparison with $W(r)$, and that

$$
\left|S_{2}\right| \leqq \sum_{Q_{1}<r \leq Q_{1}^{k-1}} \frac{\mu(r)^{2}}{\phi\left(\left|a_{0}\right|[q, r]\right)} W_{2}(r)
$$

For a prime $p$, we write $W_{2}(p)$, similarly to $W(p)$, as follows.

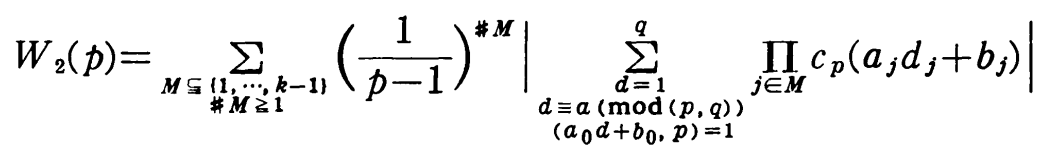

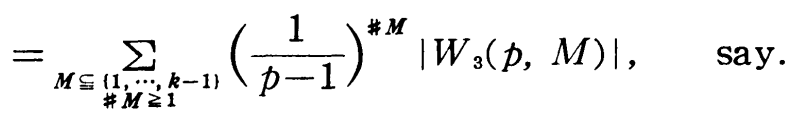

For $p \mid q$, we have $\left|W_{3}(p, M)\right| \leqq 1$ by (3.1). For $p \nmid q$, noticing that

$$
\left|\sum_{j \in M} c_{p}\left(a_{j} d+b_{j}\right)\right| \leqq 1 \quad \text { unless } \quad \prod_{j=1}^{k-1}\left(a_{j} d+b_{j}\right) \equiv 0(\bmod p),
$$

we have $\left|W_{3}(p, M)\right| \leqq k(p-1)^{\# M}+p$,

Especially, we consider the case $p \nmid\left(a_{0} q R(\boldsymbol{b})\right)$. If $\# M=1$ then

$$
W_{3}(p, M)=(p-1)+(-1)(p-2)=1 \text {, }
$$

and if $\# M \geqq 2$ then

$$
\left|W_{3}(p, M)\right| \leqq(p-1) \cdot(\# M)+1 \cdot(p-1-\# M) \leqq k p .
$$


By these evaluations and (6.7), it follows that $W_{2}(p) \ll 1$ for any prime $p$, and that $W_{2}(p) \ll 1 / p$ for $p \nmid\left(a_{0} q R(\boldsymbol{b})\right)$, where the implied constants depend only on $k$.

Now we obtain an inequality similar to (6.3) for $W_{2}(r)$ instead of $W(r)$, so the right-hand side of (6.6) contributes

$$
\ll \frac{\tau_{K}(q)}{q} \tau_{K}(R(\boldsymbol{b}))(\log x)^{-C+1},
$$

as before. Hence, (3.12) follows from (6.1), (6.5) and (6.8), and our proof of Theorem 2 is completed.

\section{References}

[1] A. Balog, The prime $k$-tuplets conjecture on average, Analytic number theory, Progress in Math. 85, Birkhäuser 1990, 47-75.

[2] P.T. Bateman and R.A. Horn, A heuristic asymptotic formula concerning the distribution of prime numbers, Math. Comp. 16 (1962), 363-367.

[3] H. Maier and C. Pomerance, Unusually large gaps between consecutive primes, Trans. Amer. Math. Soc. 322 (1990), 201-237.

[4] H. Mikawa, On prime twins in arithmetic progressions, Tsukuba J. Math. vol. 16 no. 2 (1992), 377-387.

[5] Ch.-d. Pan and Ch.-b. Pan, On estimations of trigonometric sums over primes in short intervals (III), Chin. Ann. of Math. 11 B 2 (1990), 138-147.

[6] A. Perelli, J. Pintz and S. Salerno, Bombieri's theorem in short intervals, Ann. Scuola Normale Sup. Pisa 11 (1984) 529-539.

Institute of Mathematics

University of Tsukuba

Tsukuba-city Ibaraki Pref.

305 Japan 